\title{
Thermomechanical properties and fracture of resin-bonded-sand cores - Experimental study and application in aluminium foundry
}

\author{
Claire Menet ${ }^{1, *}$, Pascal Reynaud ${ }^{1}$, Gilbert Fantozzi ${ }^{1}$, Delphine Thibault ${ }^{2}$ and Adrien Laforêt ${ }^{3}$ \\ ${ }^{1}$ MATEIS, INSA Lyon, 69621 Villeurbanne, France \\ ${ }^{2}$ MONTUPET, 60290 Laigneville, France \\ ${ }^{3}$ MONTUPET, 36130 Diors, France
}

\begin{abstract}
Sand cores are used to produce internal cavities of metallic cast parts with complex shapes like automotive cylinder heads. Foundry cores are granular materials made of sand grains aggregated with binder bridges. In the cold box coring process, the binder is a polyurethane resin. It is noteworthy that during the casting of the liquid metal, the polymer binder is seriously damaged. This kind of materials has been poorly investigated so far. This study aims for a better understanding of the mechanical behaviour and fracture of cores subjected to various loads and thermal ageing. Particularly, the focus is on the decoring step, which consists in removing the sand by hammering and vibration of the metallic part after casting. This major project, generated from the collaboration of the aluminum casting company Montupet, and two laboratories Centre des Matériaux (CdM) and MATEIS, includes both experimental and numerical activities in order to model the decoring step of cylinder heads based on empiric data. Here, the experimental part of the work is presented.
\end{abstract}

\section{Introduction}

Cold box coring process is used in foundry industry for decades. It consists of mixing silica sand with a both phenolic resin component and an isocyanate hardener in organic solvents in low amount (around 1 wt.\%). Then the mixture is injected into a permanent mould, and after catalysis, the binder is reticulated and the sand core has sufficient mechanical properties to be handled and used for the metal casting. The liquid metal (near $700^{\circ} \mathrm{C}$ in the case of aluminum alloys) is cast and then cooled at a controlled rate. The sand is finally removed during the decoring step by hammering and vibration of the whole part. Nowadays, the different parameters of the process are based on the long experience in foundry history but have not been scientifically optimized. However, the decoring step becomes ever more critical with the decreasing size of the automotive motors, which induces ever more complex shapes of the metallic parts. Indeed, sand core fragments should be evacuated through the pipes of the metallic part. A numerical modelling of this step is essential to determine parameters such as the position, the frequency, the intensity of the hammer hits, and increase development time. Before implementing such a numerical model, it is necessary to have a complete characterization of the thermo-mechanical behaviour and fracture mechanisms of sand cores, depending on the thermal ageing induced in particular by the casting and cooling temperature, the binder nature and content for example.
Granular materials with a solid binder in low proportion are not common materials and only few studies deal with foundry sand cores, and even less with their mechanical properties [1]. Some peculiar properties of granular matter have already been pointed out like the stress transfer through a network of contact chains $[2,3]$. Several papers propose experimental and/or numerical models to improve the understanding of the effects of contacts and cementation of the contacts in granular materials $[4,5,6]$. Another non-trivial phenomenon is the strain localisation developed under compression in the form of shear bands. These bands are associated with volumetric effects: dilation or contraction depending on the confining pressure (respectively low or high). Localisation has been detected in the case of both uncemented [7] and cemented [8] granular materials.
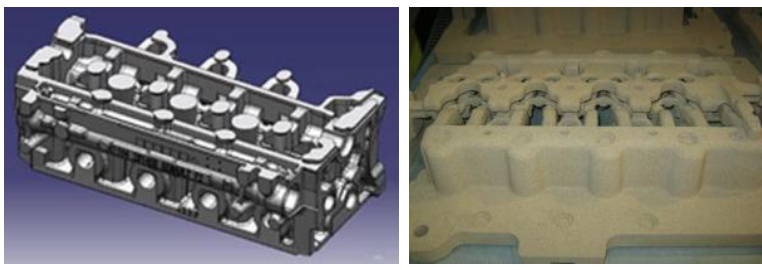

Fig. 1. An aluminum cylinder head casted with a sand casting process - Montupet (left) and the associated sand core of the bottom part of the cylinder head (right)

\footnotetext{
* Corresponding author: claire.menet@insa-lyon.fr
} 
This experimental study requires to consider the specific characteristics of sand cores: a plastic organic resin binder in low proportion, a difference in stiffness between grains and binder (that preventing grains from breaking), and particularly the thermal degradation of the binder.

\section{Materials and Methods}

Cores samples have been made in Montupet Laigneville following the industrial coring process. Silica sand grains have a rounded shape and a diameter between 50 and $250 \mu \mathrm{m}$. The commercial binder is a polyurethane resulting of the polyaddition of a formo-phenolic resin and the MDI isocyanate. Its content is lower to $1,5 \mathrm{wt} . \%$.

The mechanical response of the material is examined under uniaxial compression, 4-point bending and shear tests. Compression tests have been performed on cylindrical samples with a diameter of $30 \mathrm{~mm}$ and a height of $45 \mathrm{~mm}$. The displacement is measured with an extensometer $(25 \mathrm{~mm})$. Samples for the bending tests are $220 \mathrm{~mm}$ long bars with a section of $22.5 \times 22.5 \mathrm{~mm}^{2}$. The displacement is measured with a LVDT sensor. For the shear test, double-notched parallelepipeds are compressed. The shape of the sample is described in Fig.2. All the tests have been performed with an electromechanical testing machine (Zwick), with a controlled displacement of $0.5 \mathrm{~mm} / \mathrm{min}$. Both monotonous and cyclic tests have been performed. For the cyclic loading, a new cycle is started every $50 \mu \mathrm{m}$ of crossbar displacement.

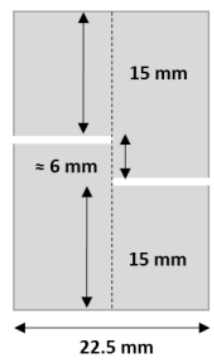

Fig. 2. Double-notched sample used for the shear test

To simulate the thermal degradation induced by the casting, some samples are aged $30 \mathrm{~min}$ in a furnace at a temperature noted $\theta$ under atmosphere conditions. The tests are performed at room temperature.

Some compressive tests have been followed by X-ray tomography. The resolution of the images is $17 \mu \mathrm{m}$. Strain fields are calculated via the image correlation software Ufreckles (developed by J. Réthoré at LaMCoS) with a finite element method.

\section{Influence of the thermal ageing}

Sand cores require specific properties because they need to be tough enough to withstand handling and the weight of the metal without deforming or breaking. However, after the casting, the cores have to lose their strength to be removed easily from the cavities. The thermal degradation of the resin binder is the key of these contradictory properties. In this following section, the effects of this degradation on the mechanical properties of the core will be investigated.

The evolution of polyurethane resin as a function of the temperature has been studied with DSC and TGA tests [9]. Two domains of weight loss appear: the first one is located between $100^{\circ} \mathrm{C}$ and $200^{\circ} \mathrm{C}$ and corresponds to an evaporation of the solvent. The second one, starts near $250^{\circ} \mathrm{C}$ and is much broader. It is linked to several oxidation reactions and finally to a pyrolysis of the resin.

\subsection{SEM observations}

The fracture surface of a core broken under bending is observed under SEM. Grains are intact, the fracture is inter-granular and occurs via the rupture of the binder bridges. Fig.3. reveals the impact of the degradation on the aspect and fracture surface of the bonds. First of all, bubbles appear from $200^{\circ} \mathrm{C}$, become ever more numerous and at $350^{\circ} \mathrm{C}$ some bridges are actually hollow. Indeed, at this temperature some torn off bridges have been noticed. These bubbles create defects into the bonds, which become weaker and moreover decrease the contact area between resin and grain. Another significant point is the differences on the fracture surfaces: the signs of plasticity (torn aspect) noticeable for the as-received sample are not anymore present for aged ones.

There is an agreement between the SEM observation and the TGA domains of degradation: damages occur from $150^{\circ} \mathrm{C}$ and get worse after $250^{\circ} \mathrm{C}$.

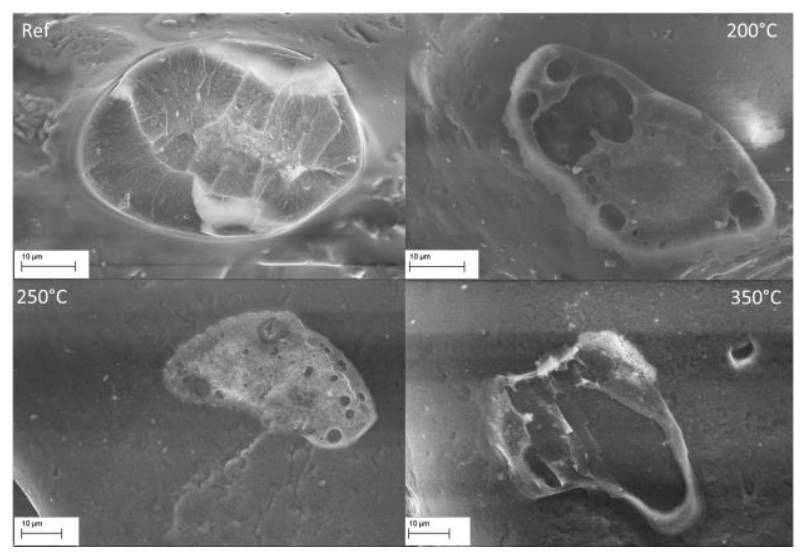

Fig. 3. Fracture surfaces of broken binder bridges depending on various ageing temperatures: unaged, $200^{\circ} \mathrm{C}, 250^{\circ} \mathrm{C}$ and $350^{\circ} \mathrm{C}$.

\subsection{Mechanical resistance}

As-received and thermally aged samples are tested under compression, bending and shear. The rupture resistances $\mathrm{R}$ under this various loads are indexed below in fig.4. $\mathrm{R}$ is defined as the peak compressive stress for 
compression tests, as the peak tensile stress in the middle point of the lower sample face for 4-point bending, and as the peak shear stress for double-notched shear test.

$$
\left\{\begin{array}{c}
R(\text { compression })=F / D \\
R(\text { bending })=\frac{3 F\left(L_{\text {inf }}-L_{\text {sup }}\right)}{2 H B^{2}} \\
R(\text { shear })=\frac{F}{h B}
\end{array}\right.
$$

With F the applied load, D the diameter of the sample, $\mathrm{H}$ the height of the sample, B the sample width, $\mathrm{L}_{\text {inf }}$ and $\mathrm{L}_{\text {sup }}$ respectively the spans between the lower and top bending supports, $\mathrm{h}$ the distance between the two notches of the shear sample (fig.2.).

Unsurprisingly, the resistance to compression is much higher than bending or shear ones because the granular skeleton supports the stresses. The low resistance under shear stress is related to the formation of shear bands in compression tests.

The evolution of the mechanical resistance follows the scheme of the degradation of the binder with a drop of the resistance from around $200^{\circ} \mathrm{C}$. A slight increase in the properties could be detected at $150^{\circ} \mathrm{C}$, it could be explained by a stiffening of the material due to the solvent evaporation and a possible continuation of the reticulation process.

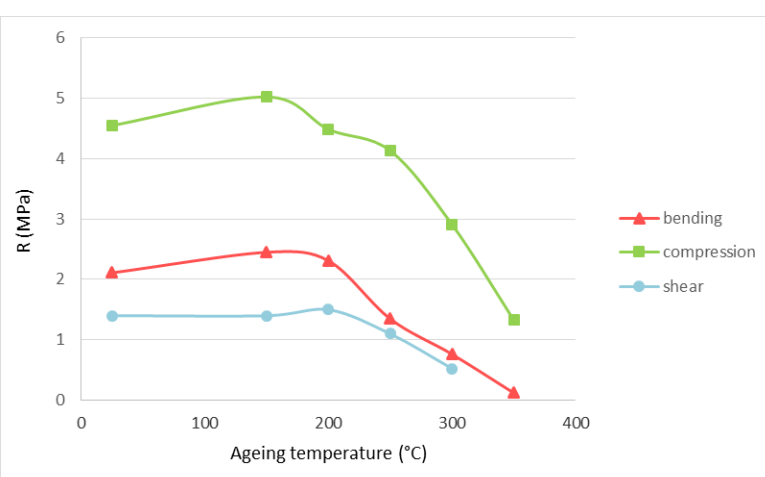

Fig. 4. Breaking resistance under bending, compression and shear as a function of $\theta$.

\subsection{Modification of the properties}

As noticed in the 3.1. section, thermal degradation not only creates bubbles which weaken the material but there is also a loss of plasticity. An example is given in Fig.5. with stress-strain curves in compression for an unaged sample and a $250^{\circ} \mathrm{C}$-aged one. If the breaking resistances are similar for the both cases, the overall behaviour is totally different.

For $\theta=250^{\circ} \mathrm{C}$, the fracture is more brittle and the plasticity is limited. Plasticity is evaluated during the load with the residual strain at the end of an unload cycle. For aged samples, the residual strain before the peak stress is halved. Secant modulus of the cycles decrease sharply after the peak stress for the aged sample, whereas it decreases linearly for unaged ones.
Likewise, the ratio of the area of the loops per the elastic energy increases a lot after the peak only in the case of aged samples. This ratio represents the dissipated energy during a cycle (friction of the grains mostly). Evolutions of secant modulus are generated by grain reorganisation, which modifies the stress-supporting network and also by eventual damages (bond ruptures). The brutal modification of the cycle characteristics after the maximal stress for aged samples may be the cause of a serious damage leading to the rupture of the sample. For unaged sample, the plasticity of the bonds permits a progressive grain reorganisation leading more slightly to the rupture.

For bending tests, the fracture is brittle for both unaged and aged samples. A really slight plasticity is detected for unaged samples, and disappears totally at $250^{\circ} \mathrm{C}$ of ageing. After an ageing at $350^{\circ} \mathrm{C}$, the material loses its resistance against tension.

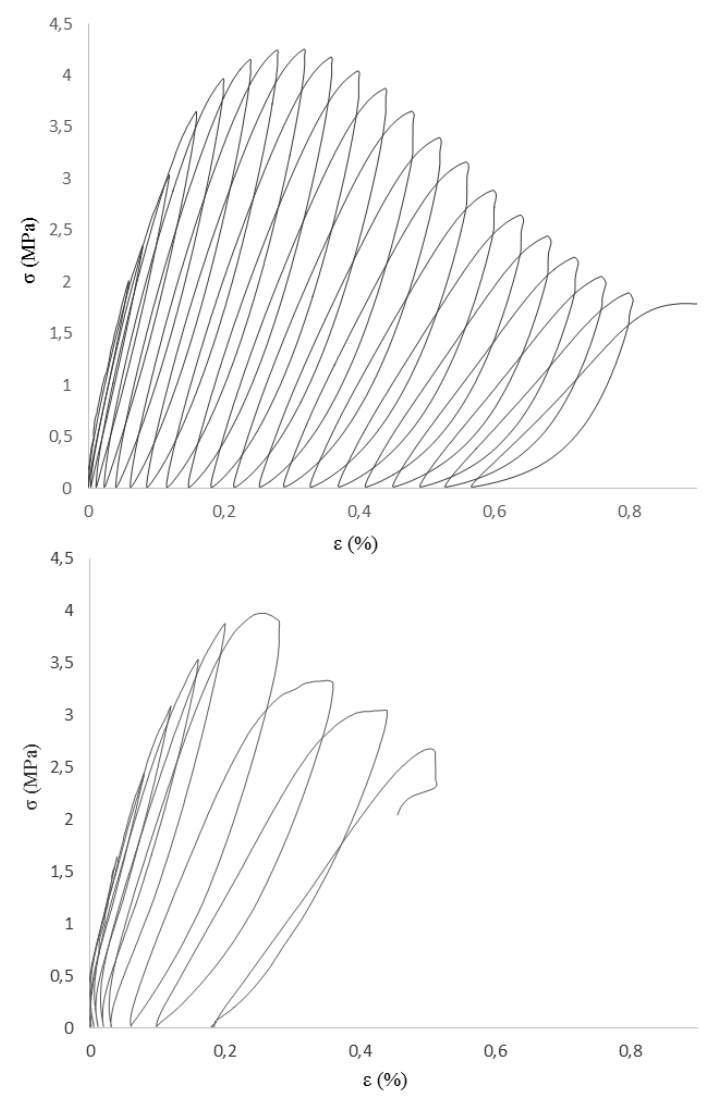

Fig. 5. Stress-strain curves for cycled compressive tests for an unaged sample (top) and a $250^{\circ} \mathrm{C}$-aged one (bottom)

\section{Fracture}

The initiation and development of cracks are really concerning issues to study the decoring process. For bending tests, fracture is generated in the tension zone. A unique crack crosses the bar: which is thin and skirts clustered grains. For information, fracture propagation was examined through a toughness assessment test on notched bars submitted to bending. This test indicates 
that the critical size of defects is in the order of the millimetre, corresponding to the size of a skirted cluster.
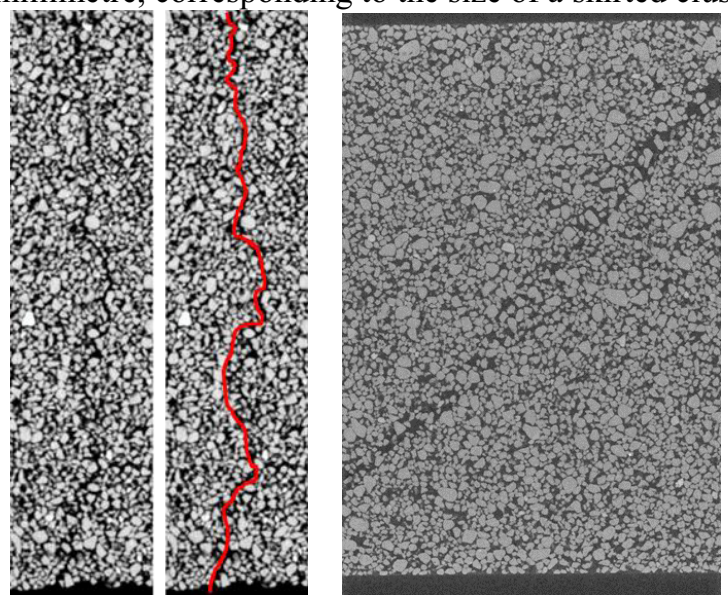

Fig. 6. Tomography stacks: (left) fracture in tension with a horizontal tensile direction; (right) shear zone in compression with a vertical compressive direction

It is common for granular materials with no/or low cementation to present localisation bands under compressive loads, and sand cores are not an exception to the rule [7,8]. A shear band with a thickness of around 4 grains could be observed in Fig. 6 .
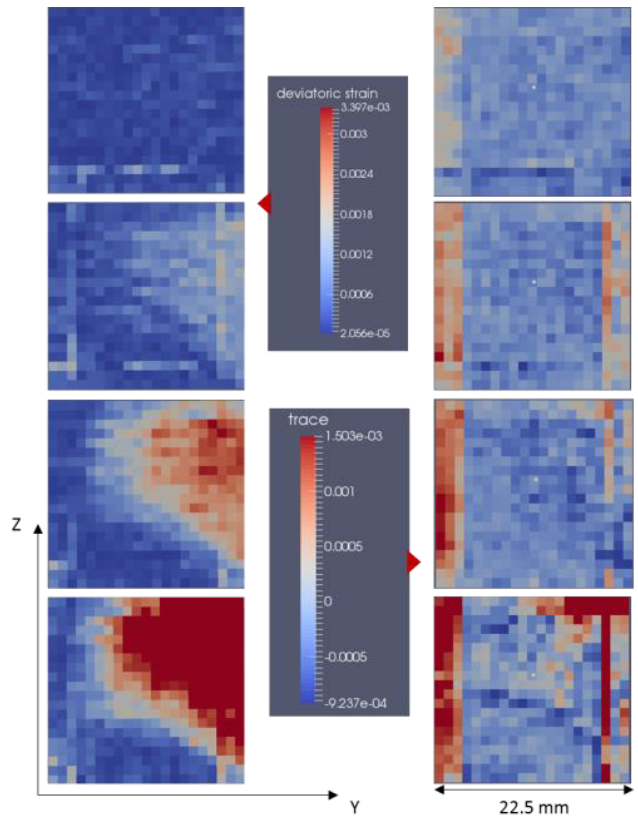

Fig.7. Deviatoric strain (left) and $3 *$ volumetric strain (right) of an unaged sample during a compressive test. The pictures correspond to an applied load of $28 \%, 56 \%, 73 \%$ and $81 \%$ of the maximal load (from top to bottom).

Some compressive in-situ tests have been performed in a $\mathrm{X}$-ray tomograph. It appears that it is difficult to distinguish cracks from pores. In fact, no crack is noticeable before the final fracture of the sample. According to literature [7-10], shear bands appear before the peak stress. The Figure 7 illustrates the deviatoric and the volumetric strain evolution during a compressive test. The sample tends to dilate on its edges (the number of binder bridges per grains is lower there). After the elastic limit, the deviatoric strain concentrates in the whole top corner, it is also possible to distinguish in this area a slight contraction followed by a slight dilation. The erosion of the structure plays an essential role for uniaxial compression.

\section{Conclusion and perspectives}

In this paper, the mechanical behaviour of sand cores has been examined through different methods. Macroscopically, monotonous and cyclic tests inform about the damages and the dissipated energy occurring during bending or compression tests. Microscopically, SEM pictures permit an analysis of the fracture surface, when in-situ tomography underlines the strain localisation. Three domains appear: an elastic one, then a plastic one, where localisation, damage and erosion start. A crack appears at the peak stress and propagates immediately for bending and monotonous compression. The fracture could be more controlled in the case of cyclic compression (stress relaxation). The originality of this study stands in the consideration of the thermal degradation of the organic binder. Correlated tests link this degradation to a loss of mechanical resistance and an embrittling. To continue the study of this material and establish a behaviour law, different results of cycled tests and creep under various loads must be gathered for samples with varying parameters: proportion of the binder, temperature of the test, and nature of the binder.

\section{Acknowledgment}

This research project has been funded and supported by the ANR agency within the frame of FIMALIPO project. Thanks are particularly due to W. Hilth and Prof. D. Ryckelynck from the CdM for fruitful discussions.

\section{References}

1. B.J. Stauder, H. Kerber, P. Schumacher, Journal of Materials Processing Technology 237 (2016)

2. A. Drescher, G. De Josselin de Jong, J. Mech. Phys. Solids 20 (1972)

3. V. Topin, J.Y. Delenne, F. Radjai, L. Brendel, F. Mabille, Eur. Phys. J. E 23 (2007)

4. J.Y. Delenne, V.Topin, F. Radjai, Acta Mechanica 205 (2009)

5. M. Jiang, W. Zhang, Y. Sun, S. Utili, Granular Matter 84 (2013)

6. N.S. Nguyen, H. Magoariec, B. Cambou, C.R. Mecanique 342 (2014)

7. J. Desrues, E. Andò, C.R. Physique 16 (2015)

8. Y.H. Wang, S.C. Leung, J. Geotech. Geoenviron. Eng. 134, 7 (2008)

9. G. Jomaa, P. Goblet, C. Coquelet., V. Morlot, Thermochimica Acta 612, (2015) 